\title{
Identifying Mycobacterium species and strain typing using a microfluidic labchip instrument
}

\author{
Robert C. Cooksey, Josef Limor, Glenn P. Morlock, and Jack T. Crawford
}

BioTechniques 35:786-794 (October 2003)

\begin{abstract}
We developed schemes for rapid identification of Mycobacterium species and strain typing using a microfluidic labchip instrument. A 439-bp region of the gene that codes for the 65-kDa heat shock protein (hsp65), which has sequence polymorphisms specific for most mycobacterial species, was examined using PCR-restriction analysis (PRA). We performed PRA in duplicate, using 2 strains each of 12 species, and observed that fragment sizes $(\mathrm{bp})$ determined automatically by the instrument were consistently smaller than the correct sizes for each of the species as determined by sequence analysis (mean variance, $<7$ bp). Mycobacterium tuberculosis isolates were typed with the labchip instrument using mycobacterial interspersed repetitive unit-variable number tandem repeat (MIRU-VNTR) typing, which determines the number of copies of repeated units at 12 loci in the genome based on product size after PCR amplification. Seven strains with one to six repeat copies at each locus were examined. Sizes were smaller by a mean of 13.47 bp compared with correct sizes predicted by sequence analysis, but could be used to correctly identify all strain types. Isolates of Mycobacterium chelonae and Mycobacterium abscessus were typed using randomly amplified polymorphic DNA (RAPD) electrophoresis, and patterns obtained using the labchip instrument were compared with multilocus enzyme electrophoresis (MEE) types. Patterns were distinct and reproducible for all strains except those with closely related MEE types. The labchip instrument is a versatile alternative for sizing mycobacterial DNA fragments.
\end{abstract}

\section{INTRODUCTION}

Accurate and expeditious laboratory identification of the species Mycobacterium tuberculosis, antimicrobial susceptibility testing, and genotyping for epidemiologic purposes contribute to the success of tuberculosis (TB) control programs. Two commercial nucleic amplification methods for detecting and identifying $M$. tuberculosis and other species in the M. tuberculosis complex in less time than by conventional methods have received approval for clinical use by the U.S. Food and Drug and Administration (1). Identification of nontuberculous mycobacteria (NTM) is also warranted, since at least 33 species have been associated with disease outbreaks (2).

Although many mycobacteriology laboratories in the U.S. currently use high-performance liquid chromatography (HPLC) of mycolic acids to identify NTM isolates (3), genotypic methods to identify polymorphisms in either $16 \mathrm{~S}$ rDNA or hsp65, which encodes the $65-\mathrm{kDa}$ heat shock protein using restriction analyses, have been described (4-7). The standardized method for PCR restriction analysis (PRA) of $h s p 65$ involves determining the sizes of restriction fragments after a 439-bp PCR product is digested with BstEII or HaeIII (8). Since the sizes of these fragments can be estimated only when they are electrophoresed in agarose or acrylamide gels and compared with a sizing standard included in the gel, additional methods are needed for DNA electrophoresis, in which the sizes of fragments are automatically and accurately determined.

The control of TB and other mycobacterial diseases also relies on laboratory characterizations of the relationships among isolates that may be involved in disease outbreaks. The application of the insertion sequence IS6110 as a probe to evaluate heterogeneities in the genomes of M. tuberculosis isolates have advanced TB control efforts throughout the world. Newer genotypic methods, including spacer oligonucleotide typing (spoligotyping) (9) and mycobacterial interspersed repetitive unit-variable number tandem repeat (MIRU-VNTR) typing (10) are currently under evaluation as more efficient alternatives to IS6110 fingerprinting for typing $M$. tuberculosis isolates. The latter of these methods does not require DNA-DNA hybridizations and is a simple amplification assay to determine the number of tandem nucleotide repeats based on PCR product sizes at 12 regions in the M. tuberculosis genome. Like $h s p 65$ PRA, accurate determination of the sizes of MIRU-VNTR amplimers after electrophoresis is required. A multiplex assay enabled by the use of fluorescently labeled primers and high-throughput analysis including automatic product sizing was described in 2001 as a means to reduce the number of individual PCRs from 12 and to remove subjectivity in determining the sizes of PCR products (11).

The need for expeditious epidemiologic typing of NTM organisms has intensified in parallel with the increasing 
frequency of reported outbreaks caused by these species. Typing methods that have demonstrated applicability to most, or all, NTM species include multilocus enzyme electrophoresis (MEE) using starch gels (12), pulse-field gel electrophoresis of infrequent-target restriction enzyme digests (13), and randomly amplified polymorphic DNA (RAPD) electrophoresis (14). The last of these methods offers the greatest ease of performance, since it is also a simple PCR procedure using a single short (10-mer) oligonucleotide and low (e.g., $37^{\circ} \mathrm{C}$ ) annealing temperatures. Determination of product sizes after electrophoresis is not critical for RAPD typing, but patterns of isolates can be compared only with those included in the same gel. Isolates with patterns that match in the numbers and electrophoretic mobilities of bands are considered to be a common strain.

This assortment of genotypic assays to characterize mycobacteria that have electrophoresis of linear doublestranded DNA (dsDNA) in common demonstrates the need for a universal performance format. As for most modern laboratory technology, the features of such an electrophoresis format should include low cost, ease and speed of use, accuracy, reproducibility, versatility, and low waste generation. Microfluidic devices are part of recent technology that may rank high in each of these categories (15).

We evaluated a microfluidic labchip instrument, the Model 2100 Bioanalyzer (Agilent Technologies, Palo Alto, CA, USA), for performance of PRA to identify Mycobacterium species, for MIRU-VNTR typing of $M$. tuberculosis isolates, and for RAPD typing of NTM isolates. A sieving polymer containing a fluorescent dye is distributed in microchannels of a disposable labchip using manual pressure applied with a syringe. Twelve samples $(1 \mu \mathrm{L}$ each) and a molecular-size ladder in individual wells move through the microchannels and are then injected into a separation channel where components are electrophoretically separated and detected by fluorescence. A typical analytical run requires approximately $30 \mathrm{~min}$, and data are displayed as both simulated bands on gel images and peaks in electropherograms. The in- strument is conveniently small $(162 \times$ $412 \times 290 \mathrm{~mm}$ ) and is complemented with an assortment of kits, including disposable labchips for flow cytometric, RNA, and protein analyses, as well as analyses of dsDNA in four size groups (25-500 bp; 25-1000 bp; 100-7500 bp; and 100-12,000 bp). The manufacturer states that the accuracy of size determinations by the instrument may err by between $10 \%$ and $15 \%$, depending on the kit used, and states that quantitation of dsDNA in a range of 0.5-50 ng may err by $\leq 30 \%$. We customized protocols for performance of each assay using the labchip instrument and evaluated results for accuracy and reproducibility.

\section{MATERIALS AND METHODS}

\section{Bacterial Strains}

Seven strains of M. tuberculosis and 22 isolates representing 11 NTM species were obtained from our stock collection. The M. tuberculosis strains were typed using the MIRU-VNTR method (10). Strains were selected on the basis of diversity at each of the 12 loci as determined previously. These strains (and their MIRU-VNTR patterns) were 2837 (125325143224), 2833 (224225143225), $1467 \quad$ (223324163633), $1473 \quad(2343$ 25153321), 2832 (322325163643), 2844 (233325153322), and $\mathrm{H}_{37} \mathrm{Rv}$ (ATCC27294) (ATCC, Manassas, VA, USA) (22.53226133321). The last of these strains has an aberrant copy number between 2 and 3 at the second MIRU region (no. 4) (16).

In addition to M. tuberculosis, 2 isolates each of 11 additional species were selected and identified previously by mycolic acid patterns using HPLC (3). These species (and isolates) included Mycobacterium abscessus (99-6081 and 99-6127), Mycobacterium avium (TMC721 and ATX3A), Mycobacterium chelonae (99-6141 and 99-6090), Mycobacterium fortuitum (85-1247 and 85-1112), Mycobacterium gordonae (88-688 and 88-703), Mycobacterium phlei (TMC1548 and TMC1516), Mycobacterium scrofulaceum (TMC1320 and TMC1305), Mycobacterium simiae (TMC1226 and 85-1137), Mycobacterium szulgai (Jenkens 6555 and Wolinsky 465), Mycobacterium 
Table 1. Restriction Analyses of hsp65 of 12 Mycobacterium Species Using the Model 2100 Bioanalyzer

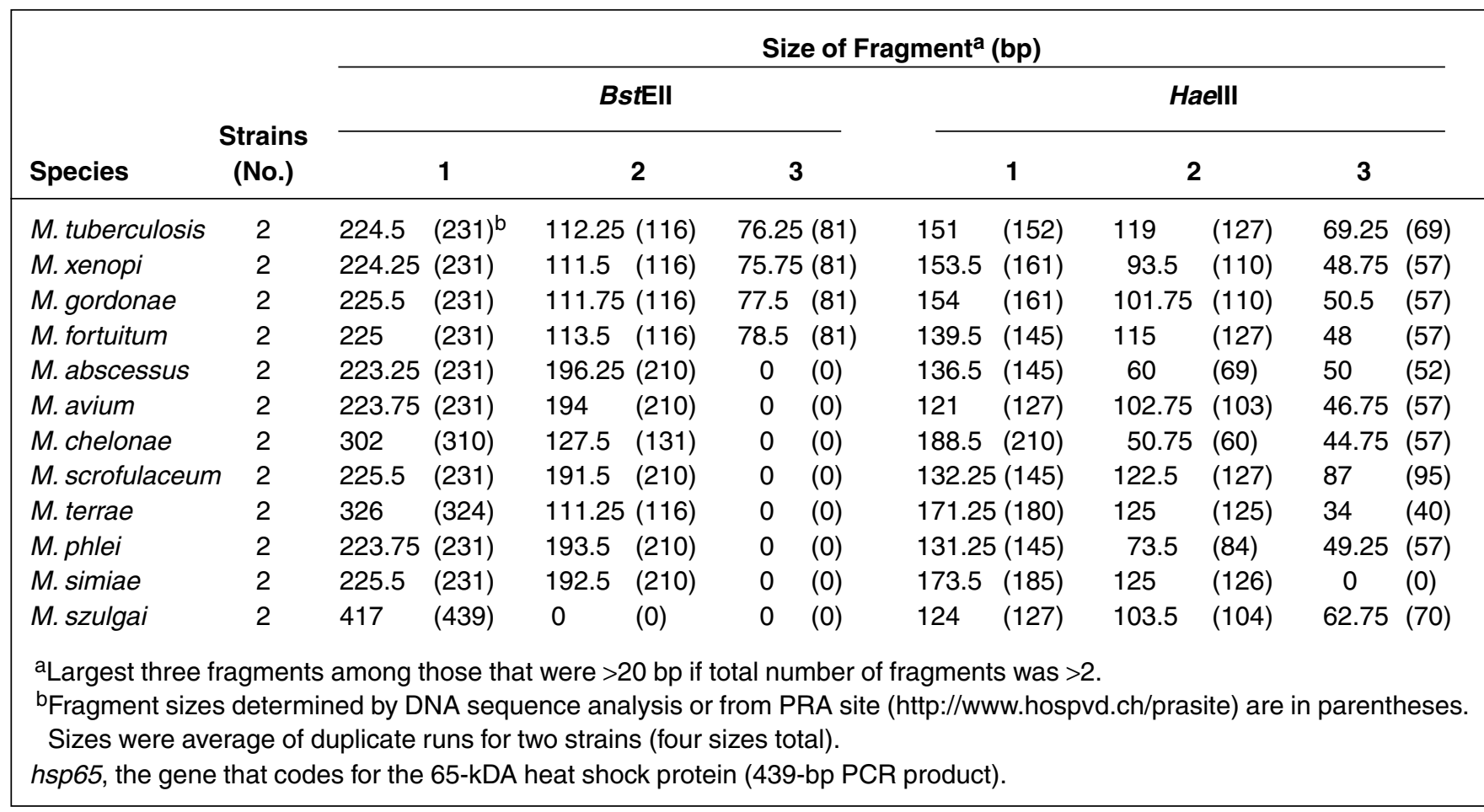

terrae (ATCC15755 and ATCC25213), and Mycobacterium xenopi (TMC1482 and 91-TI47). The two isolates each of M. abscessus and M. chelonae were tested along with eight additional $M$. abscessus isolates using the RAPD typing method described below. The relationships of these isolates were previously evaluated using MEE (17). The 10 M. abscessus isolates (and their MEE patterns) included 99-6175 (type 1), 99-6202 (type 2), 99-6029 (type 3), 99-6044 (type 4), 99-6052 (type 5), 99-6081 (type 6), 99-6127 (type 11), and 99-6137 (type 14), 99-6068 (type 15 ), and 99-6050 (type 18), and the two M. chelonae isolates included 99-6141 (type 22) and 99-6090 (type 23).

\section{Genotypic Methods}

Crude DNA preparations were obtained from Middlebrook 7H9 broth cultures (Remel, Lenexa, KS, USA) grown at $37^{\circ} \mathrm{C}$ (except for $M$. fortuitum, M. abscessus, and $M$. chelonae, which were incubated at $30^{\circ} \mathrm{C}$ ) to stationary growth phase using a bead agitation method (18) with the following modifications: $1 \mathrm{~mL}$ of culture was added to a 2-mL BioPulverizer tube prepackaged with Lysing Matrix B
(Qbiogene, Carlsbad, CA, USA). Cells were disrupted using a FastPrep ${ }^{\circledR}$ Model FP120A agitator (Qbiogene) for $40 \mathrm{~s}$ at a power setting of 6 . For PRA, these crude DNA templates $(1 \mu \mathrm{L})$ were used in PCRs to amplify a 439-bp region of the gene that codes for the $65-\mathrm{kDa}$ heat shock protein (hsp65) (19) using primers TB11 and TB12 (8) and conditions as previously described (20), except that the annealing temperature during PCR was $60^{\circ} \mathrm{C}$. Restriction endonucleases (HaeIII and BstEII) were obtained from Sigma (St. Louis, MO, USA) and used to digest the hsp 65 products as previously described (21).

Typing of $M$. tuberculosis strains was performed by the MIRU-VNTR method using 12 primer sets, the nucleotide sequences of which are available upon request. Reaction mixtures containing $5 \mu \mathrm{L}$ of HotStarTaq ${ }^{\mathrm{TM}}$ DNA Polymerase Master Mix (Qiagen, Valencia, CA, USA) and $0.4 \mu \mathrm{L}(200 \mathrm{nM})$ of each primer, in a total volume of 9 $\mu \mathrm{L}$, were added to $1 \mu \mathrm{L}$ template DNA. PCR amplification was performed using a GeneAmp ${ }^{\circledR}$ PCR System 9700 Thermal Cycler (Applied Biosystems, Foster City, CA, USA) set for the following conditions: $15 \mathrm{~min}$ at $95^{\circ} \mathrm{C}, 40$ cycles of $30 \mathrm{~s}$ at $94^{\circ} \mathrm{C}, 30 \mathrm{~s}$ at $65^{\circ} \mathrm{C}$, and $1 \mathrm{~min}$ at $72^{\circ} \mathrm{C}$, with a final incubation for $7 \mathrm{~min}$ at $72^{\circ} \mathrm{C}$.

The RAPD typing method was performed as previously described (22). Primers were 10-mer oligonucleotides and included RAPD1 (22), OPC2, OPC5, OPC8, OPA2, and OPA7 $(23,24)$. Reaction mixtures for RAPD PCR were prepared as described above, except that only a single primer was used in each reaction at a final concentration of 600 nM. The stability of RAPD patterns (i.e., the number and electrophoretic mobility of gel bands) as related to the age of cultures was evaluated by extracting DNA templates from 1-mL aliquots of broth cultures incubated for 5, 7, 9, 12,14 , or 16 days. PCR amplification was performed as described above, except that only 35 cycles were used, and the annealing step was $1 \mathrm{~min}$ at $37^{\circ} \mathrm{C}$.

\section{Electrophoresis}

Aliquots of PRA, MIRU, and RAPD reactions were electrophoresed in both conventional horizontal agarose gels and in the microfluidic labchip instrument. Restriction digests $(5 \mu \mathrm{L})$ were electrophoresed at $100 \mathrm{~V}$ for $2 \mathrm{~h}$ in $3 \%$ Metaphor agarose gels (BioWhittaker Molecular Applications, Rockland, 
ME, USA) prepared in $1 \times$ Tris-borate EDTA (TBE). The MIRU and RAPD aliquots $(5 \mu \mathrm{L})$ were electrophoresed in $2 \%$ Metaphor gels using the same conditions. Metaphor gels were incubated for 30-60 min at $4^{\circ} \mathrm{C}$ prior to running, as recommended by the manufacturer. MIRU products were prepared and analyzed in duplicate. Restriction digests were treated with an equal volume of phenol/chloroform/isoamyl alcohol (PCI) $(50: 49: 1, \mathrm{v} / \mathrm{v})$. The samples were briefly vortex mixed after the PCI was added, the aqueous (upper) layer was retrieved after microcentrifugation for $10 \mathrm{~min}$ at $3400 \times g$, and $1 \mu \mathrm{L}$ was analyzed on the labchip instrument using the DNA 500 and DNA 1000 LabChip ${ }^{\circledR}$ kits (Agilent Technologies) according to the manufacturer's instructions. The same volumes $(1 \mu \mathrm{L})$ of MIRU and RAPD preparations were analyzed on the labchip instrument using DNA 1000 and DNA 7500 kits, respectively. Samples were combined in labchip wells with a gel matrix containing dye and internal size standards included with each of the kits, vortex mixed, and electrophoresed. In addition to a simulated gel image and chromatogram, the labchip instrument software also provided tabulated results that included band migration time (s), sizes (bp), concentration (ng/ $\mu \mathrm{L})$, corrected peak area, and molarity (nmol/ L) of DNA fragments. The DNA fragment sizes were evaluated for the PRA and MIRU procedures, but not for the

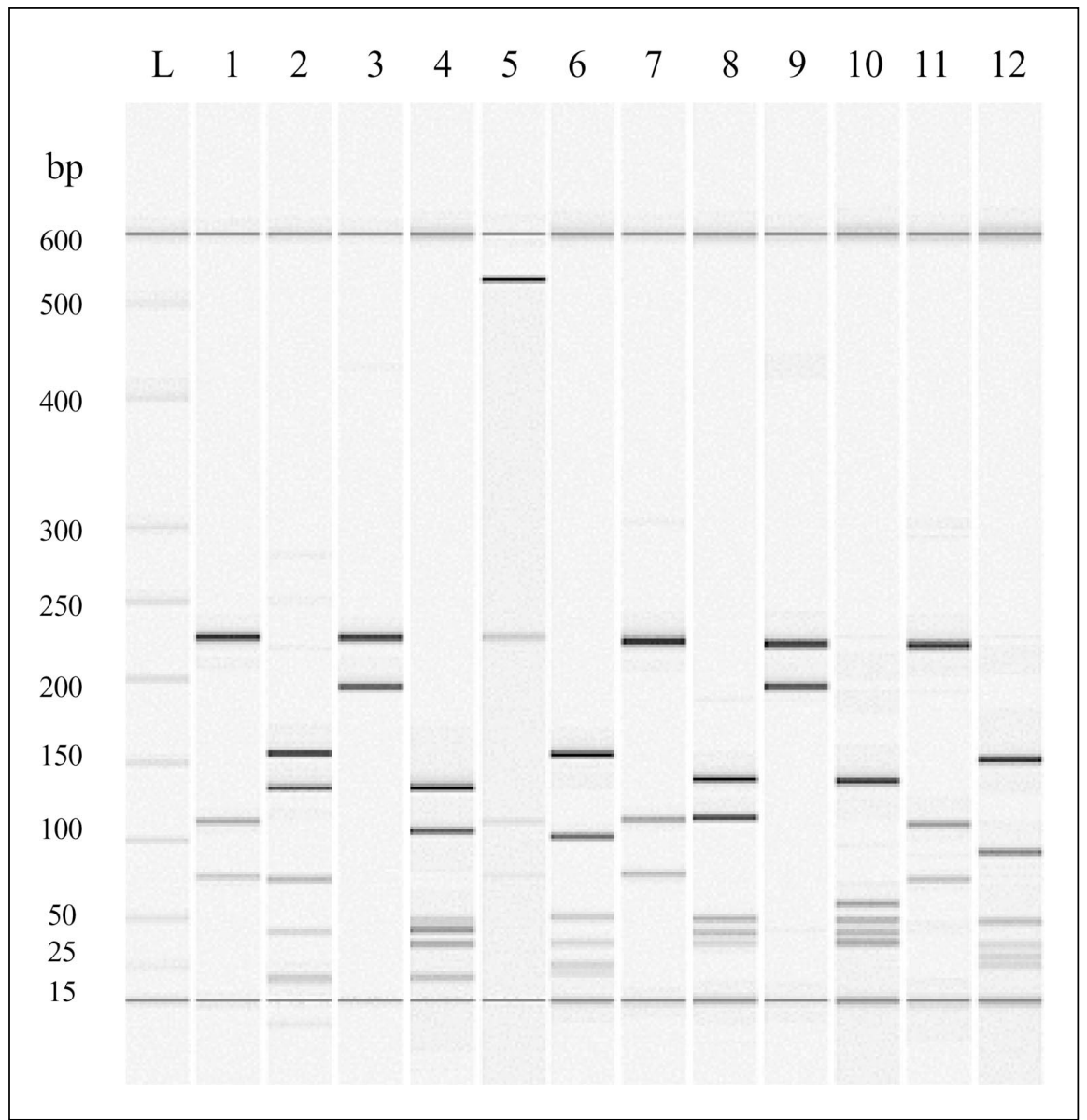

Figure 1. PCR restriction analysis of the gene that codes for the 65-kDa heat shock protein (hsp65). The 439-bp hsp65 PCR products were digested with either BstEII or HaeIII (followed by treatment with phenol/chloroform/isoamyl alcohol; 50:49:1, v/v), and $1 \mu \mathrm{L}$ was tested on the labchip instrument using the manufacturer's DNA 500 kit. The size standard is in lane L with fragment sizes (bp) listed on the left, and internal size markers for lane normalization align with 15- and 600-bp ladder bands. BstEII digests are in lanes 1, 3, 5, 7, 9, and 11, and HaeIII digests are in lanes 2, 4, 6, 8, 10, and 12. Isolates included Mycobacterium tuberculosis $\mathrm{H}_{37} \mathrm{Rv}$ (lanes 1 and 2), Mycobacterium avium TMC721 (lanes 3 and 4), Mycobacterium gordonae 88-688 (lanes 5 and 6), Mycobacterium fortuitum 85-1247 (lanes 7 and 8), Mycobacterium abscessus 99-6081 (lanes 9 and 10), and Mycobacterium xenopi TMC1482 (lanes 11 and 12). 
RAPD results for which major bands in each of the lanes on the simulated gel image were compared.

\section{RESULTS}

Two isolates each of 12 Mycobacterium species were examined by $h s p 65$ PRA. The 439-bp hsp65 PCR products were digested with BstEII and HaeIII in separate reactions, and $1 \mu \mathrm{L}$ of each was examined on the Model 2100 Bioanalyzer using the DNA 500 kit. The HaeIII patterns were uninterpretable, because the two internal size standards were absent after electrophoresis, most likely because of restriction by residual HaeIII despite heating the reactions to $65^{\circ} \mathrm{C}$ for $10 \mathrm{~min}$ to inactivate the endonuclease activity. The treatment with PCI eliminated the apparent degradation of the internal size standards, and interpretation of fragment sizes was restored to the labchip instrument. The internal standards were apparently unaffected by residual enzyme activity in $B s t$ EII digests, and patterns were interpretable without additional treatment of the restriction digests. The predicted sizes of restriction fragments (Table 1) were obtained either from a multicenter Web site for $h s p 65$ PRA analyses (http: //www.hospvd.ch/prasite) or from the locations of restriction sites using our own sequence data (19). The largest three fragments generated by $B s t$ EII and Hae III that were $>20$ bp were evaluated for each of the 12 species except for those species for which less than three fragments were obtained. These patterns included six restriction fragments for four species, five fragments for six species, and four fragments for two species (Table 1). The sizes of restriction fragments that were determined by the labchip instrument are shown in Table 1 as the average of four results (duplicate runs for the two isolates of each species). These averages differed from the predicted fragment sizes in a range of 2 bp greater to $22 \mathrm{bp}$ smaller, and the average variance was -6.53 bp (Table 1). Restriction patterns for the $M$. xenopi and M. gordonae isolates matched. An electrophoretic gel image for six isolates is shown in Figure 1 and resembled patterns observed after 5- $\mu \mathrm{L}$ samples of digests were electrophoresed in $3 \%$ Metaphor agarose gels. The BstEII digest of M. gordonae isolate 88-688 (lane 5) was apparently incomplete, since the predominant band identified by the labchip instrument correlated with undigested PCR product even though three additional bands consistent with the BstEII pattern for M. gordonae were also identified.

Twelve MIRU-VNTR regions for each of seven M. tuberculosis strains were examined by electrophoresis of PCR products on the labchip instrument using the DNA 1000 kit (Table 2). The MIRU-VNTR types were confirmed

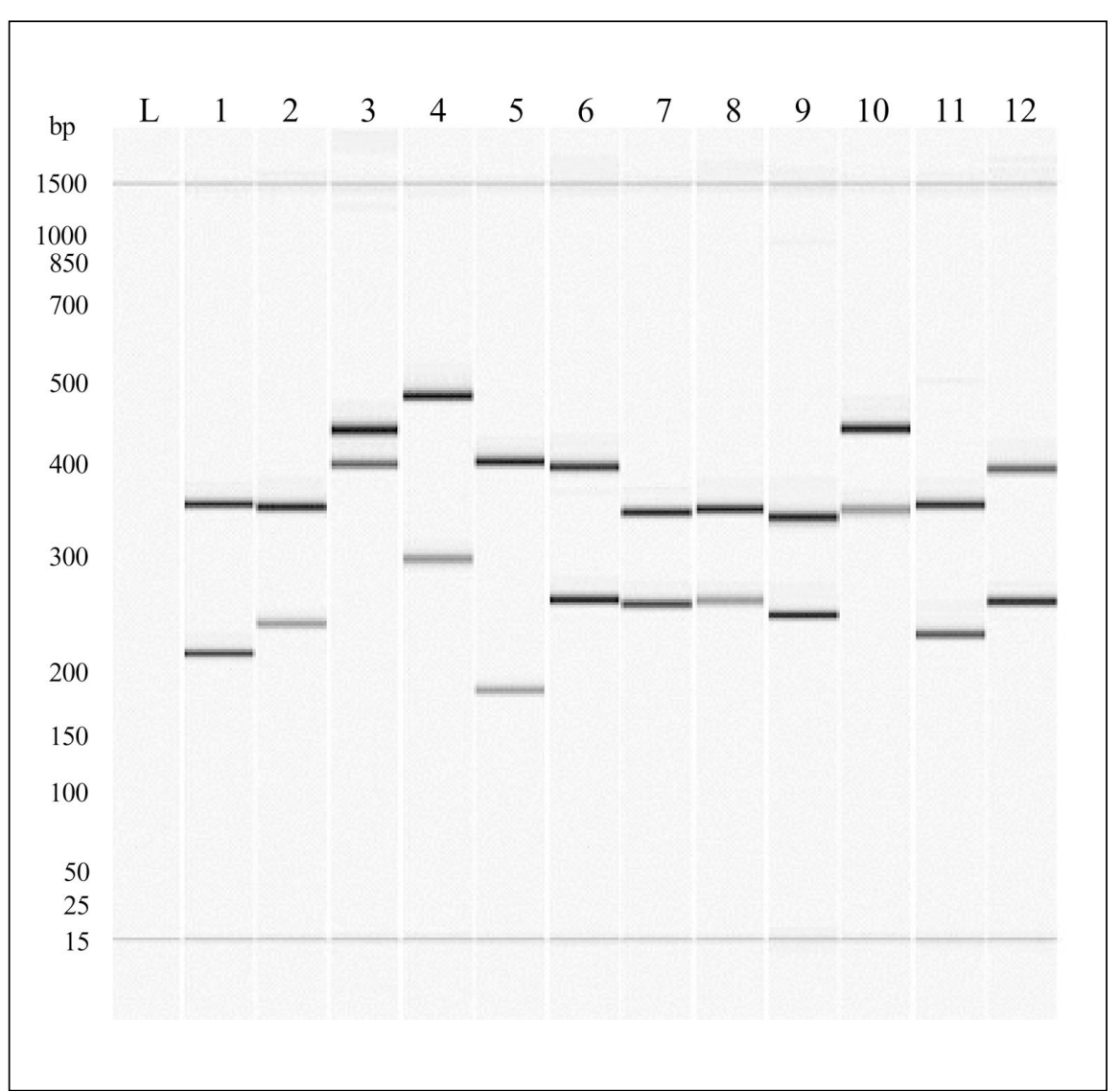

Figure 2. Duplex mycobacterial interspersed repetitive unit (MIRU) analyses of 2 Mycobacterium tuberculosis strains. Strain 2837 is in lanes $1-6$, and $\mathrm{H}_{37} \mathrm{Rv}$ is in lanes 7-12. MIRU regions examined were 2 and 24 (lanes 1 and 7), 4 and 20 (lanes 2 and 8), 10 and 40 (lanes 3 and 9), 16 and 23 (lanes 4 and 10), 26 and 31 (lanes 5 and 11), and 27 and 39 (lanes 6 and 12). Internal size standards are shown as uppermost and lowermost bands in each lane. 
by electrophoresing $5-\mu \mathrm{L}$ aliquots of each of the 12 PCRs in $2 \%$ Metaphor agarose gels. The number of MIRU repeats for each region ranged from 1 to 6 copies, and 32 MIRU products were represented among the 12 regions. The sizes of all products determined by the labchip instrument were less than those predicted from sequence analyses and ranged from $-6.6 \%$ (MIRU-VNTR locus no. 23 with 6 copies of the repeat) to $-1.9 \%$ (locus no. 2 with 1 copy).

The average variance of 166 results from predicted values was $13.47 \mathrm{bp}$ $(-3.63 \%)$. Duplex MIRU analysis was based on the selection of six pairs of MIRU regions for which none of the possible products for one region were of the same size as any possible product for the second region and for which only two specific products were observed for each of the seven strains. The average variance was used to estimate possible PCR product sizes for regions for which we had no strains to represent the number of repeat copies. An example was MIRU-VNTR region no.10, for which our seven test isolates had either $2,3,4$, or 5 copies of the 53-bp repeat (Table 2). The predicted product size for a theoretical strain having only a single copy of the repeat is $273 \mathrm{bp}$, and the predicted result for the labchip instrument is $3.63 \%$ smaller, or approximately $263 \mathrm{bp}$. Regions that were paired according to the absence of matching products based on observed or predicted sizes included regions 2 and 24 , 4 and 20, 10 and 40, 16 and 23, 26 and 31 , and 27 and 39. One microliter of the products was examined for strains 2837 and $\mathrm{H}_{37} \mathrm{Rv}$ using the labchip instrument, and two bands were observed in each lane (Figure 2). Sizes of duplex MIRU products were within the ranges shown in Table 2.

Among the six primers evaluated for RAPD typing of $M$. abscessus and $M$. chelonae isolates, PCR using primer RAPD1 yielded more electrophoretic bands and better differentiation among strains as determined by MEE patterns than did PCR using the remaining five primers. The RAPD patterns of $M$. abscessus and $M$. chelonae isolates that represented $12 \mathrm{MEE}$ types that were electrophoresed using the labchip instrument are shown in Figure 3. The RAPD patterns for all isolates were different except for those seen for $M$. abscessus MEE types 4, 5, and 6, and minor patterns differences were noted between MEE types 11 and 14 .

Although band sizes that were determined by the labchip instrument were not routinely evaluated for RAPD experiments, none of the five most intense bands differed by $1 \%$ or more in size among MEE type isolates 4, 5, and 6 or among MEE types 11 and 14. The same RAPD pattern relationships were observed using the remaining 5 primers, even though some interpretations proved to be more difficult because of the paucity of bands for these primers. Banding patterns represented by the labchip instrument's gel image were generally easier to interpret than those observed after analyzing $5-\mu \mathrm{L}$ RAPD samples in 2\% Metaphor gels, since nonspecific lane smears and curved bands were less apparent. The reproducibility of RAPD patterns as related to the age of broth cultures used to prepare DNA templates was evaluated for strain 99-6052 (MEE type 5) using primer RAPD1. No differences in patterns were observed using $1-\mathrm{mL}$ aliquots of broth cultures harvested after incubation for $5,7,9,12,14$, or 16 days of incubation (data not shown).

\section{DISCUSSION}

The analyzed results of data obtained using the labchip instrument agreed with results obtained from the same samples using conventional agarose electrophoresis or by HPLC analyses for species identification. The 24 isolates representing 12 Mycobacterium species were correctly identified by PRA, as were the MIRU-VNTR patterns of $7 \mathrm{M}$. tuberculosis strains despite errors in results that varied from predicted sizes by an average of 6.53 bp for PRA and $13.47 \mathrm{bp}$ for MIRUVNTR. With only rare exception, the sizes of fragments and PCR products were smaller than the predicted sizes, but the overall error was less than that warned by the manufacturer (10\%-15\%). Although these average variances will most likely change if additional strains are examined, the existing data have enabled our design of interpretive tables for both species identification and MIRU-VNTR types. 
Table 2. Evaluation of MIRUs for Seven Mycobacterium tuberculosis Isolates Using the Labchip Instrument

\begin{tabular}{|c|c|c|c|c|c|c|c|c|}
\hline MIRU-VNTR & $\begin{array}{l}\text { Repeat } \\
\text { Size } \\
\text { (bp) }\end{array}$ & $\begin{array}{l}\text { Repeat } \\
\text { Copies }^{a}\end{array}$ & Strains $^{b}$ & $\begin{array}{l}\text { Predicted } \\
\text { PCR } \\
\text { Product } \\
\text { (bp) }\end{array}$ & $\begin{array}{c}\text { Results } \\
\text { (No.) }\end{array}$ & $\begin{array}{l}\text { Observed } \\
\text { Range } \\
\text { (bp) }\end{array}$ & $\begin{array}{l}\text { Observed } \\
\text { Mean } \\
\text { (bp) }\end{array}$ & $\begin{array}{c}\text { Mean } \\
\text { Variance } \\
(\%)\end{array}$ \\
\hline \multirow[t]{3}{*}{2} & 53 & 1 & 1 & 238 & 2 & $232-235$ & 233.5 & -1.9 \\
\hline & & 2 & $2,3,4,5,7$ & 291 & 10 & $275-286$ & 284.1 & -2.7 \\
\hline & & 3 & 6 & 344 & 2 & $335-336$ & 335.5 & -2.5 \\
\hline \multirow[t]{2}{*}{$4^{c}$} & 77 & 2 & $1,2,3,6$ & 268 & 8 & $256-263$ & 260.8 & -2.7 \\
\hline & & 3 & 4,7 & 345 & 4 & $334-338$ & 336.75 & -2.4 \\
\hline \multirow[t]{4}{*}{10} & 53 & 2 & 6 & 326 & 2 & $315-316$ & 315.5 & -3.3 \\
\hline & & 3 & $3,5,7$ & 379 & 6 & $355-368$ & 364.8 & -3.7 \\
\hline & & 4 & 2,4 & 432 & 4 & $416-421$ & 418.25 & -3.2 \\
\hline & & 5 & 1 & 485 & 2 & $452-471$ & 461.5 & -4.8 \\
\hline \multirow[t]{2}{*}{16} & 53 & 2 & $2,5,7$ & 475 & 6 & $454-463$ & 461.17 & -2.9 \\
\hline & & 3 & $1,3,4,6$ & 528 & 8 & $496-517$ & 512.5 & -2.9 \\
\hline 20 & 77 & 2 & $1-7$ & 375 & 14 & $362-369$ & 366.9 & -2.2 \\
\hline \multirow[t]{3}{*}{23} & 53 & 4 & 3 & 289 & 2 & $274-276$ & 275.0 & -4.8 \\
\hline & & 5 & $1,2,4,6,7$ & 342 & 10 & $315-337$ & 327.4 & -4.3 \\
\hline & & 6 & 5 & 395 & 2 & $362-376$ & 369.0 & -6.6 \\
\hline 24 & 52 & 1 & $1-7$ & 386 & 14 & $359-377$ & 374.57 & -3.0 \\
\hline \multirow[t]{4}{*}{26} & 51 & 3 & 5 & 399 & 2 & $366-393$ & 379.5 & -4.9 \\
\hline & & 4 & 1,2 & 450 & 4 & $411-430$ & 424.0 & -5.8 \\
\hline & & 5 & 4,7 & 501 & 4 & $478-482$ & 480.25 & -4.2 \\
\hline & & 6 & 3,6 & 552 & 4 & $528-533$ & 530.25 & -4.0 \\
\hline 27 & 53 & 3 & $1-7$ & 436 & 14 & $401-425$ & 420.36 & -3.6 \\
\hline \multirow[t]{3}{*}{31} & 53 & 2 & 1,2 & 215 & 4 & $201-211$ & 207.5 & -3.5 \\
\hline & & 3 & $4,5,7$ & 268 & 6 & 249-261 & 257.5 & -3.9 \\
\hline & & 6 & 3,6 & 427 & 4 & $416-420$ & 418.5 & -4.1 \\
\hline \multirow[t]{3}{*}{39} & 53 & 2 & $1,2,4,5,7$ & 297 & 10 & $276-288$ & 284.6 & -4.2 \\
\hline & & 3 & 3 & 350 & 2 & $333-337$ & 335.0 & -4.3 \\
\hline & & 4 & 6 & 403 & 2 & $386-390$ & 388.0 & -3.7 \\
\hline \multirow[t]{5}{*}{40} & 54 & 1 & 4,5 & 284 & 4 & $265-271$ & 268.25 & -5.5 \\
\hline & & 2 & 7 & 338 & 2 & $320-322$ & 321.0 & -5.0 \\
\hline & & 3 & 3,6 & 392 & 4 & $370-373$ & 371.0 & -5.4 \\
\hline & & 4 & 1 & 446 & 2 & $407-425$ & 416.0 & -6.7 \\
\hline & & 5 & 2 & 500 & 2 & $472-476$ & 474.0 & -5.2 \\
\hline $\begin{array}{l}\text { aAmong all isol } \\
\text { bStrains include } \\
\text { cAberrant copy } \\
\text { MIRU-VNTR, m }\end{array}$ & $\begin{array}{l}\text { es in study } \\
2837(1), \\
\text { umber for } \\
\text { cobacteria }\end{array}$ & $\begin{array}{l}\text { set. } \\
2833 \text { (2), } 14 \\
\text { ocus no. } 4 \text { c } \\
\text { interspers }\end{array}$ & $\begin{array}{l}67 \text { (3), } 1473 \text { ( } \\
\text { strain } \mathrm{H}_{37} \mathrm{Rv} \\
\text { d repetitive ur }\end{array}$ & $\begin{array}{l}\mathrm{H}_{37} \mathrm{Rv}(5) \text {, } \\
\text { 6) not consi } \\
\text { variable nur }\end{array}$ & $\begin{array}{l}2(6) \text {, anc } \\
\text { ed. } \\
r \text { tandem }\end{array}$ & $4(7)$ & & \\
\hline
\end{tabular}


Sizes of the largest fragments $(\leq 3$ fragments) for each restriction enzyme used for PRA were reduced by the average variance, assigned an additional range of $\pm 5 \%$ to allow for flexibility in observed data, and used to develop a Microsoft Excel7 algorithm to identify 44 Mycobacterium species or PRA subtypes of species. We likewise used our MIRU-VNTR data or product sizes that were reduced by the average variance to create interpretive tables for MIRU-VNTR analyses performed using the labchip instrument. Since all 12 wells of each disposable labchip used in the Model 2100 Bioanalyzer are required for MIRU-VNTR analysis of one M. tuberculosis isolate (or two isolates if duplex MIRU-VNTR PCR is performed), the instrument cannot be regarded as high-throughput. Twoprimer MIRU-VNTR typing of a set of
12 isolates, for example, would require as many labchips and a minimum of 6 $\mathrm{h}$ after PCR in addition to preparation time. The introduction of a similar microfluidic instrument with expanded capability (e.g., simultaneous analyses of 96 rather than 12 samples) could reduce turnaround times as well as cost per sample.

At least nine RAPD patterns were present among 10 MEE groups of $M$. abscessus and 2 MEE groups of $M$. chelonae examined. The MEE groups for which matching RAPD patterns were found (MEE types 4, 5, and 6) were previously shown to be similar by phylogenetic analysis (genetic distance, $<0.07$ ) (17). Although the fragments were sized and quantitated by the labchip instrument software, emphasis was placed on analyses of bands with the greatest intensities as shown on the simulated gel

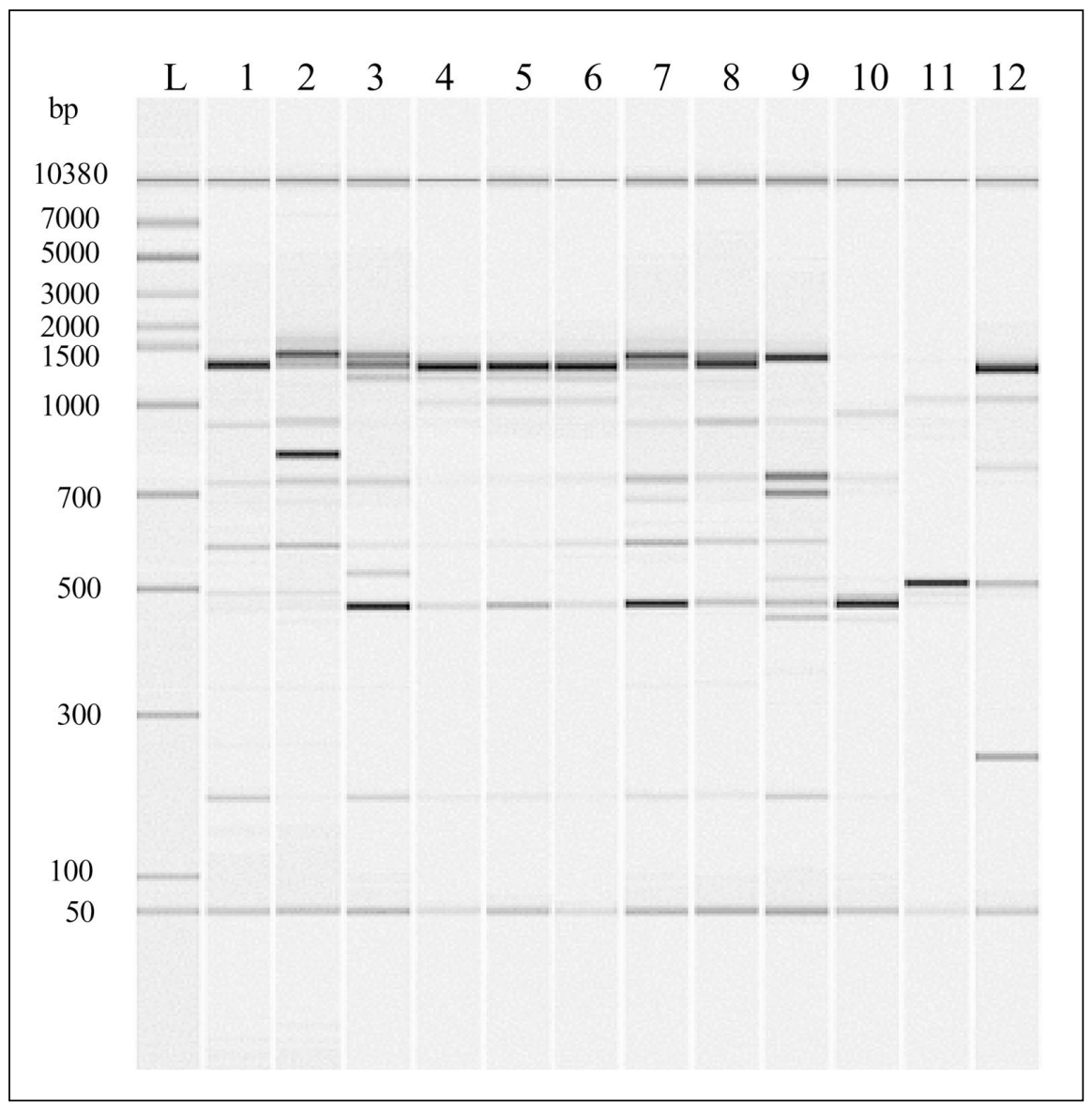

Figure 3. Randomly amplified polymorphic DNA (RAPD) patterns of 10 Mycobacterium abscessus and two Mycobacterium chelonae isolates. PCR products were prepared using primer RAPD1 (22) and analyzed using the DNA 7500 kit. Isolates of M. abscessus (lanes 1-9) and M. chelonae (lanes 10-12) were typed using multilocus enzyme electrophoresis (MEE) typing (17). These MEE types for M. abscessus isolates were 1 (lane 1), 2 (lane 2), 3 (lane 3), 4 (lane 4), 5 (lane 5), 6 (lane 6), 11 (lane 7), 14 (lane 8), 15 (lane 9), and 18 (lane 10). The MEE types for M. chelonae isolates were 22 (lane 11) and 23 (lane 12). 
image. These patterns overall were easier to interpret than the same patterns after electrophoresis in 2\% Metaphor agarose gels. Among the six previously described RAPD primers that we used to evaluate $M$. chelonae and M. abscessus isolates, RAPD1 provided optimal strain resolution and will, therefore, be the primer of choice in future evaluations of these species. Our intention, however, is to continue to evaluate all six RAPD primers against other NTM species.

Instructions for using the labchip instrument were straightforward, and a minimum of training was required. Each of the three identification and typing schemes we evaluated using the labchip instrument utilize the same DNA template, and the volumes of reagents may be reduced since only $1 \mu \mathrm{L}$ is evaluated in the instrument. These may be considered to be additional cost-saving factors.

Our findings are not meant to address the validity of the three procedures used in this study, but rather to determine if the Model 2100 Bioanalyzer is a suitable adjunct format for their performance. Even though errors in electrophoretic data were observed using the labchip instrument, sizes of DNA products from duplicate runs were generally reproducible and resulted in correct interpretations of species identifications and strain types.

\section{ACKNOWLEDGMENTS}

We thank Dr. Lauren S. Cowan of the Centers for Disease Control and Prevention Tuberculosis/Mycobacteriology Branch for valuable assistance in performance of MIRU-VNTR typing of $\mathrm{M}$. tuberculosis isolates and for help in the design of the computer algorithm. Please note that use of commercial trade names is for descriptive purposes only and does not constitute endorsement by the U.S. Department of Health and Human Services, The Public Health Service, or the Centers for Disease Control and Prevention.

\section{REFERENCES}

1.Woods, G.L. 2002 The mycobacteriology laboratory and new diagnostic techniques. Infect. Dis. Clin. North Am. 16:1-15.
2.Wallace, R.J., Jr., B.A. Brown, and D.E. Griffith. 1998. Nosocomial outbreaks/pseudo outbreaks caused by nontuberculous mycobacteria. Ann. Rev. Microbiol. 52:453-490.

3.HPLC Users Group. 1996. Standardized Method for HPLC Identification of Mycobacteria. Centers for Disease Control and Prevention, Atlanta, GA.

4.Avaniss-Aghajani, E., K. Jones, A. Holtzman, T. Aronson, N. Glover, M. Boian, S. Froman, and C. Brunk. 1996. Molecular technique for rapid identification of mycobacteria. J. Clin. Microbiol. 34:98-102.

5.Kirschner, P., B. Springer, U. Vogel, A. Meier, A. Wrede, M. Kiekenbeck, F.C. Bange, and E.C. Bottger. 1993. Genotypic identification of mycobacteria by nucleic acid sequence determination: report of a 2-year experience in a clinical laboratory. J. Clin. Microbiol. 31:2882-2889.

6.Plikaytis, B.B., B.D. Plikaytis, M.A. Yakrus, W.R. Butler, C.L. Woodley, V. Silcox, and T.M. Shinnick. 1992. Differentiation of slowly growing Mycobacterium species, including Mycobacterium tuberculosis, by gene amplification and restriction fragment length polymorphism analysis. J. Clin. Microbiol. 30:1815-1822.

7.Springer, B., L. Stockman, K. Teschner, G.D. Roberts, and E.C. Bottger. 1996. Two-laboratory collaborative study on identification of mycobacteria: molecular versus phenotypic methods. J. Clin. Microbiol. 34: 296-303.

8.Telenti, A., F. Marchesi, M. Balz, F. Bally, E.C. Bottger, and T. Bodmer. 1993. Rapid identification of mycobacteria to the species level by polymerase chain reaction and restriction enzyme analysis. J. Clin. Microbiol. 31:175-178

9.Kamerbeek, J., L. Schouls, A. Kolk, M. van Agterveld, D. van Soolingen, S. Kuijper, A. Bunschoten, H. Molhuizen, et al. 1997. Simultaneous detection and strain differentiation of Mycobacterium tuberculosis for diagnosis and epidemiology. J. Clin. Microbiol. 35:907-914.

10.Supply, P., E. Mazars, S. Lesjean, V. Vincent, B. Gicquel, and C. Locht. 2000. Variable human mini-satellite-like regions in the Mycobacterium tuberculosis genome. Mol. Microbiol. 36:762-771.

11.Supply, P., S. Lesjean, S. Savine, K. Kremer, D. van Soolingen, and C. Locht. 2001. Automated high-throughput genotyping for study of global epidemiology of Mycobacterium tuberculosis based on mycobacterial interspersed repetitive units. J. Clin. Microbiol. 39:3563-3571

12.Selander, R.K., D.A. Caugant, H. Ochman, J.M. Musser, M.N. Gilmour, and T.S. Whittam. 1986. Methods of multilocus enzyme electrophoresis for bacterial population genetics and systematics. Appl. Environ. Microbiol. 51:873-884.

13.Mazurek, G.H., S. Hartman, Y. Zhang, B.A. Brown, J.S.R. Hector, D. Murphy, and R.J. Wallace, Jr. 1993. Large DNA restriction fragment polymorphism in the Mycobacterium avium-M. intracellulare complex: a potential epidemiologic tool. J. Clin. Microbiol. 31:390-394.
14.Welsh, J. and M. McClelland.1990. Fingerprinting genomes using PCR with arbitrary primers. Nucleic Acids Res. 18:7213-7218.

15.Fitzgerald, D.A. 2002. Riding the microfluidic wave. Scientist 16:40-43.

16.Cowan, L.S., L. Mosher, L. Diem, J.P. Massey, and J.T. Crawford. 2002. Variablenumber tandem repeat typing of Mycobacterium tuberculosis with low copy numbers of IS6110 by using mycobacterial interspersed repetitive units. J. Clin. Microbiol. 40:15921602.

17.Yakrus, M.A., S.M. Hernandez, M.M. Floyd, D. Sikes, W.R. Butler, and B. Metchock. 2001. Comparison of methods for identification of Mycobacterium abscessus and $M$. chelonae isolates. J. Clin. Microbiol. 39:4103-4110.

18.Plikaytis, B.B., R.H. Gelber, and T.M. Shinnick. 1990. Rapid and sensitive detection of Mycobacterium leprae using a nested-primer gene amplification assay. J. Clin. Microbiol. 28:1913-1917.

19.Shinnick, T.M. 1987. The 65-kilodalton antigen of Mycobacterium tuberculosis. J. Bacteriol. 169:1080-1088.

20.Cooksey, R.C., G.P. Morlock, B.P. Holloway, J. Limor, and M. Hepburn. 2002. Temperature-mediated heteroduplex analysis performed by using denaturing high-performance liquid chromatography to identify sequence polymorphisms in Mycobacterium tuberculosis complex organisms. J. Clin. Microbiol. 40:1610-1616.

21.Hernandez, S.M., G.P. Morlock, W.R. Butler, J.T. Crawford, and R.C. Cooksey. 1999. Identification of Mycobacterium species by PCR-restriction fragment length polymorphism analyses using fluorescence capillary electrophoresis. J. Clin. Microbiol. 37:3688-3692.

22.Zhang, Q., R. Kennon, M.A. Koza, K. Hulten, and J.E. Clarridge III. 2002. Pseudoepidemic due to a unique strain of $\mathrm{Myco}$ bacterium szulgai: genotypic, phenotypic, and epidemiological analysis. J. Clin. Microbiol. 40:1134-1139.

23.Kauppinen, J., R. Mantyjarvi, and M.-L. Katila. 1994. Random amplified polymorphic DNA genotyping of Mycobacterium malmoense. J. Clin. Microbiol. 32:1827-1829.

24.Kauppinen, J., E.-L. Hintikka, E. Iivanainen, and M.-L. Katila. 2001. PCR-based typing of Mycobacterium avium isolates in an epidemic among farmed lesser white-fronted geese (Anser erythropus) Vet. Microbiol. 81: 41-50.

Received 14 May 2003; accepted 13 June 2003.

\section{Address correspondence to:}

Robert C. Cooksey

Tuberculosis/Mycobacteriology Branch

Centers for Disease Control and Prevention

Mail stop F-08

Atlanta, GA 30333,

e-mail: rccl@cdc.gov 